\title{
A PRECONDITIONING MASS MATRIX TO AVOID THE ILL-POSED TWO-FLUID MODEL
}

\author{
Angel L. Zanotti, Carlos G. Méndez, Norberto M. Nigro and Mario Storti \\ International Center for Computational Methods in Engineering (CIMEC) \\ INTEC-Universidad Nacional del Litoral-CONICET, S3000GLN \\ Güemes 3450, Santa Fe, Argentina \\ Tel.: 54-342-4511594, Fax: 54-342-4550944, e-mail: azanotti@intec.unl.edu.ar
}

\begin{abstract}
Two-fluid models are central to the simulation of transport processes in two-phase homogenized systems. Even though this physical model has been widely accepted, an inherently non-hyperbolic and non-conservative ill-posed problem arises from the mathematical point of view. It has been demonstrated that this drawback occurs even for a very simplified model, i.e., an inviscid model with no interfacial terms. Lots of efforts have been made to remedy this anomaly and in the literature two different types of approaches can be found. On one hand, extra terms with physical origin are added to model the interphase interaction, but even though this methodology seems to be realistic, several extra parameters arise from each added term with the associated difficulty in their estimation. On the other
\end{abstract}


hand, mathematical based-work has been done to find the way to remove the complex eigenvalues obtained with two-fluid model equations. Preconditioned systems, characterized as a projection of the complex eigenvalues over the real axis, may be one of the choices.

The aim of this paper is to introduce a simple and novel mathematical strategy based on the application of a preconditioning mass matrix that circumvents the drawback caused by the non-hyperbolic behavior of the original model. Although the mass and momentum conservation equations are modified, the target of this methodology is to present another way to reach a steady state solution (using a time marching scheme), greatly valued by researchers in industrial process design. Attaining this goal is possible because only the temporal term is affected by the preconditioner. The obtained matrix has two parameters that correct the non-hyperbolic behavior of the model: the first one modifies the eigenvalues removing their imaginary part and the second one recovers the real part of the original eigenvalues. Besides the theoretical development of the preconditioning matrix, several numerical results are presented to show the validity of the method.

JAM-05-1230; Zanotti, Méndez, Nigro and Storti; [2]. 


\section{INTRODUCTION}

A multi-phase flow denotes a continuum where more than a single phase is present, e.g., gas bubbles rising in a liquid, droplets of fluid moving in a gas, steam-water flows in a boiler, pipeline transport of gas, and oil mixtures and oil-gas-water flows in an oil well. These examples are found in a great variety of industrial and technological applications such as chemical reactors, boilers, combustion chambers, steelmaking plants, and nuclear power plants devices. According to the geometry of the interface, a two-phase flow can be classified into three types (see Ishii [1]): separated flows, transitional or mixed flows, and dispersed flows, with significant differences in their behavior.

When modeling a biphasic flow, it is necessary to know what phenomena, effects and flow structures are important. In some cases, the exact structure or position of the interface is important, while in other cases only some kind of average quantity is needed for the flow analysis.

Models for two-phase flows can be categorized into two different groups. The first group is the so called interface tracking models (ITM), which track the interface between the two phases, and are well suited for separated flows. The most frequently employed Eulerian-based ITM for predicting certain classes of multiphase flows are the volume of fluid (VOF) method [2-7], the front tracking (FT) or immersed boundary method [8,9], the level set (LS) methods [10-13] and the phase field (PF) methods [14-16].

Ideally, one would like to track the interface between the phases at all times, which is similar to solving all relevant scales of turbulent single-phase flow (DNS). However, this is often computationally too expensive and sometimes redundant for dispersed flows. Thus, in 
the second group of models, the exact position of the interface is not followed accurately and only spatial distributions of volume-averaged quantities such as the void fraction and the velocity field for the dispersed phases are calculated. Dispersed flows are usually modeled using models from this second group.

Two generic approaches can be used for modeling dispersed flows: the Lagrangian and the Eulerian formulation. For particulate (or particle-like) flows, it is possible to build methods based on the Marker and Cell (MAC) scheme (see Harlow and Welch [17]). The general idea is to follow each particle of the dispersed phase as they are transported by the continuous phase. This approach, in which the continuous phase is calculated in an Eulerian reference frame, is referred to as a Lagrangian-Eulerian formulation.

A different way of modeling dispersed flows is to treat both phases as a continuum. This is generally referred to as the Eulerian-Eulerian formulation or the two-fluid model. In this model, discussed in detail by Ishii [1], each phase has its own set of balance equations and the interaction between phases is represented via interface transfer terms that arise from the constitutive relations. Therefore, both phases are present in each point of the domain, each one with an associated volume fraction.

In this study, the two-fluid model is chosen to simulate dispersed biphasic flows and a mathematical analysis of this model is presented in the next sections. It is well known (see Drew and Passman [18]) that in a mathematical sense this model is ill posed because its hyperbolic nature may not be warranted for all the flow parameters. Furthermore, the model is questionable for its non-conservative formulation [19] and non-linear terms make the problem more complex for the analysis. 
Numerical solutions of ill-posed two-fluid problems have two drawbacks: excessive numerical diffusion, and instabilities. These situations are common for ill-posed initial boundary value problems (see, e.g., Joseph and Saut [20]) and, therefore, the success of any method depends on the requirement that such systems be well posed.

It is important to make a distinction between non-hyperbolic problems considered in the present work and another class of problems that address loss of hyperbolicity due to a change of type in the partial differential equations. Change of type problems are a separate subject of mathematical analysis, which is related to how the information propagates between the hyperbolic and elliptic domains [20].

A lot of effort has been made to remedy the non-hyperbolic anomaly of the two-fluid model and two different approaches can be found. One is based on the physics of the problem and consists in adding extra terms to the interfacial interaction, searching thus for a physical solution of the non-hyperbolic behavior. Even though this methodology seems to be realistic, several extra parameters arise from each added term with the associated difficulty in their estimation [21]. One of the usually added terms, which produces significant improvement in the parameter range when the problem is well posed, is the interfacial pressure. This enhanced model relaxes the assumption of equal pressure for both phases and the introduction of two pressures is justified saying that the pressure of the continuous phase, computed by the twofluid model, is far away from the not-resolved flow around a bubble. This seems logical since the two-fluid model aims are not to solve the details of the flow close to individual bubbles. In this sense, Lahey [22] has reached very promising results for air-water systems finding wellposedness for all void fractions with very large densities ratio using as a necessary condition 
that $\mathrm{Cp}>0.166$ (pressure coefficient in the pressure jump at interface). However, this lower bound may be in conflict with the results obtained by Drew and Passman [18] for highly viscous flow, where they found that $\mathrm{Cp}$ should be negative. Other contributions using different pressures for each phase that allows to extend the range of parameters for which the problem is hyperbolic are available in the literature (see, e.g., Chung et al. [23], Micaelli [24], Bestion [25], Coquel et al. [26], Lee et al. [27], Pokharna et al. [28]).

Another important and different contribution has been made by Stadtke et al. [29]. In their work, they split the interfacial momentum coupling terms in a viscous and non-viscous part. Drag forces are representative for the former. For the latter, a series of terms have been introduced in order to compensate the information lost during the averaging procedure. These terms contain only space and time derivatives of major dependent parameters, including phasic velocities, void fraction, and phasic densities, using only one pressure. The authors enumerate the criteria for the design of the model and finally demonstrate that they achieve a full set of real eigenvalues with a complete set of independent eigenvectors, warranting that the problem will be well posed [30].

From the mathematical point of view, the target is to find at least the way to remove the complex eigenvalues obtained with the two-fluid conservation equations. Following Hadamard [31], a well-posed problem is defined in these terms: "In order for a problem involving a PDE to be well-posed, the solution to the problem must exist and be unique, and solution must depend continuously upon the initial and boundary data". An equivalent definition, more suitable for numerical evaluation, may be found through the strong hyperbolic character of a given equation system. The two-fluid model is a first order in time 
system of equations with first and second order spatial derivatives for the convective and diffusive terms respectively. In addition, there are some zero order terms for sources coming from interfacial terms. Transforming these equations into a first order system, a necessary requirement for the well-posedness of the problem, is that this first order system be strongly hyperbolic (see Reula [32], Gundlach [33]), which means that the system be diagonalizable with real eigenvalues. The equivalent first order differential equations represent the time evolution of the system and the non-guaranteed real eigenvalues suffice to prove the lack of causality of the solution and the final blow up. This means that in the effort of getting a numerical solution through a time marching scheme, fatal instabilities appear and forbid not only the computation of the time evolution of the variables but also the knowledge of the steady state of the system. Even though real problems have a temporal evolution, the knowledge of the steady solution is very useful for the industrial design of processes with multiphase flow involved. This fact has motivated the idea of recovering at least the steady solution trying to modify the time marching scheme by a time preconditioner.

Preconditioned systems, characterized as a projection of the complex eigenvalues over the real axis, may be one of the possible choices. The main disadvantage of this alternative is that the original equations should be modified and so they lose some conservation properties. The present paper introduces a preconditioning mass matrix method that makes the two-fluid model hyperbolic, recovering the conservation properties for the final solution and providing an alternative to obtain steady solutions for multiphase flow problems, which will be of help for process design. 
The next sections are organized as follows: Section 2 presents the two-fluid model for a one-dimensional problem with equal phase pressures. Section 3 develops a characteristic analysis of this particular problem (see Jones and Prosperetti [34]) with emphasis on the lack of real eigenvalues in order to motivate the proposal of a preconditioning mass matrix. The paper then presents the mass matrix used as a preconditioner, analyzing the role of the two parameters included in its definition: the first one, $\beta$, is for making the model hyperbolic, and the second one, $\gamma$, is used for recovering the propagation wave speed corresponding to the original model. Finally, representative problems are solved numerically with the purpose of checking that the preconditioning method circumvents the instabilities showed by most multifluid models when using a large density ratio, high void fractions, and large relative velocities among the phases.

\section{TWO-FLUID MODEL}

The complex nature of multi-phase flows, characterized by changes in the geometrical configuration of the different phases, makes it extremely difficult to find models that reproduce the physics of the system and that are at the same time numerically tractable at a reasonable computational cost. Mathematical models based on averaged fields of the phases (see, e.g., Ishii [1], Drew and Passman [18], Zhang and Prosperetti [35]) associated with experimental correlations seem to be one of the best alternatives. These multi-field models are widely used to model and to simulate the transport of multiphase flow systems. They treat each phase as an interpenetrating continuum (field), and conservation laws are applied to each one of them. In this approximation, constitutive laws have to be provided to represent the 
interaction between fields. In Fig. 1 we can see a schematic representation of two-fluid models.

Two-phase flow averaged equations are presented below (see Ishii [1], Lahey and Drew [36]), which have been obtained as a result of temporal and/or space averaging of the instantaneous local balance equations. Without loss of generality, the simplest model proposed by Drew and Passman [18] is considered. In this one-dimensional model the flow is assumed to be inviscid, isothermal, and without phase change. Supposing for concreteness that one phase is gaseous and the other one liquid, we call $\alpha_{g}$ and $\alpha_{l}$ their corresponding volume fractions. Thus, mass and momentum balance equations can be written in the following way:

$$
\begin{gathered}
\frac{\partial\left(\alpha_{g} \rho_{g}\right)}{\partial t}+\frac{\partial\left(\alpha_{g} \rho_{g} v_{g}\right)}{\partial x}=0, \\
\frac{\partial\left(\alpha_{l} \rho_{l}\right)}{\partial t}+\frac{\partial\left(\alpha_{l} \rho_{l} v_{l}\right)}{\partial x}=0 . \\
\alpha_{g} \rho_{g} \frac{\partial v_{g}}{\partial t}+\alpha_{g} \rho_{g} v_{g} \frac{\partial v_{g}}{\partial x}=-\alpha_{g} \frac{\partial p}{\partial x}+\alpha_{g} \rho_{g} g-F_{I}, \\
\alpha_{l} \rho_{l} \frac{\partial v_{l}}{\partial t}+\alpha_{l} \rho_{l} v_{l} \frac{\partial v_{l}}{\partial x}=-\alpha_{l} \frac{\partial p}{\partial x}+\alpha_{l} \rho_{l} g+F_{I} .
\end{gathered}
$$

where $\rho, v$ and $p$ denote density, velocity and pressure, respectively. In this model, the pressure $p$ is assumed the same for both phases. Terms containing $g$ represent the gravitational force, and $F_{I}$ models the interaction between the phases. Several physical effects 
may be included in interphase force $F_{I}$ [21], but certainly the most common of them is the drag force. For dispersed flows this force can be modeled as [37]

$$
F_{I}=\frac{3}{8} C_{d} \rho_{l}\left(v_{g}-v_{l}\right)\left|v_{g}-v_{l}\right| \frac{\alpha_{g}}{r_{b}},
$$

where $r_{b}$ is the mean radius of the dispersed phase and $C_{d}$ is the drag coefficient for which empirical correlations are available as a function of the Reynolds number.

\section{CHARACTERISTICS ANALYSIS}

Examining the characteristic values of the governing equations, it can be determined if a model is properly formulated. Taking into account the constraint $\alpha_{g}+\alpha_{l}=1$, we can define $\Phi=\left(\alpha_{g}, p, v_{g}, v_{l}\right)$ as the unknown state vector and Eqs. (1)-(4) can be written in vector form as

$$
\mathbf{A} \frac{\partial \Phi}{\partial t}+\mathbf{B} \frac{\partial \Phi}{\partial x}+\mathbf{C}=0
$$

If incompressibility of each phase is assumed, matrices $\mathbf{A}, \mathbf{B}$ and $\mathbf{C}$ are

$$
\mathbf{A}=\left(\begin{array}{cccc}
1 & 0 & 0 & 0 \\
-1 & 0 & 0 & 0 \\
0 & 0 & \alpha_{g} \rho_{g} & 0 \\
0 & 0 & 0 & \left(1-\alpha_{g}\right) \rho_{l}
\end{array}\right)
$$




$$
\mathbf{B}=\left(\begin{array}{cccc}
v_{g} & 0 & \alpha_{g} & 0 \\
-v_{l} & 0 & 0 & 1-\alpha_{g} \\
0 & \alpha_{g} & \alpha_{g} \rho_{g} v_{g} & 0 \\
0 & 1-\alpha_{g} & 0 & \left(1-\alpha_{g}\right) \rho_{l} v_{l}
\end{array}\right)
$$

and

$$
\mathbf{C}=\left(\begin{array}{c}
0 \\
0 \\
-\alpha_{g} \rho_{g} g+F_{I} \\
-\left(1-\alpha_{g}\right) \rho_{l} g-F_{I}
\end{array}\right)
$$

The local linear dynamic character of Eq. (6) can be examined by linearizing the system about an initial state $\Phi_{0}$ (from now on, we assume that all matrices and derivatives are evaluated in this state). The linear differential equation for the behavior of a perturbation $\delta \Phi=\Phi-\Phi_{0}$ is

$$
\mathbf{A} \frac{\partial \delta \Phi}{\partial t}+\mathbf{B} \frac{\partial \delta \Phi}{\partial x}+\left(\frac{\partial \mathbf{A}}{\partial \Phi} \cdot \frac{\partial \Phi}{\partial t}+\frac{\partial \mathbf{B}}{\partial \Phi} \cdot \frac{\partial \Phi}{\partial x}+\frac{\partial \mathbf{C}}{\partial \Phi}\right) \delta \Phi=0
$$

A solution in the form of a traveling wave is assumed

$$
\delta \Phi=\delta \Phi_{0} \exp [i(k x-\omega t)]
$$

where $\delta \Phi_{0}$ represents the initial amplitude of the perturbation. The imaginary part $\omega_{I}$ of $\omega$ will govern growth or decay depending on its sign and the real part $\omega_{R}$ yields the speed of 
propagation. Substituting Eq. (11) into Eq. (10), the compatibility condition that $\delta \Phi_{0}$ must satisfy is

$$
-i \omega \mathbf{A} \delta \Phi_{0}+i k \mathbf{B} \delta \Phi_{0}+\left(\frac{\partial \mathbf{A}}{\partial \Phi} \cdot \frac{\partial \Phi}{\partial t}+\frac{\partial \mathbf{B}}{\partial \Phi} \cdot \frac{\partial \Phi}{\partial x}+\frac{\partial \mathbf{C}}{\partial \Phi}\right) \delta \Phi_{0}=0
$$

For an initial uniform steady state $\partial \Phi / \partial t$ and $\partial \Phi / \partial x$ are zero. Defining $\lambda=\omega / k$ and $\mathbf{D}=\partial \mathbf{C} / \partial \Phi$, the condition under which nontrivial solutions for $\delta \Phi_{0}$ exist is given by

$$
\operatorname{det}\left(\mathbf{A} \lambda-\mathbf{B}+\frac{i}{k} \mathbf{D}\right)=0
$$

In the limit as $k \rightarrow \infty$, Eq. (13) reduces to the characteristic equation corresponding to Eq. (6)

$$
\operatorname{det}(\mathbf{A} \lambda-\mathbf{B})=0
$$

and the values that $\lambda$ can take are the characteristic values. Note that algebraic terms like gravitational or drag forces, which do not contain derivatives of the unknowns, do not affect the characteristic analysis.

The characteristic values of the simplest two-fluid model, Eqs. (6)-(9), are given by

$$
\lambda=\left\{\infty, \infty, \frac{1}{d}\left(r \pm s^{1 / 2}\right)\right\}
$$

where 


$$
\begin{gathered}
r=\alpha_{g} \rho_{l} v_{l}+\left(1-\alpha_{g}\right) \rho_{g} v_{g}, \\
s=-\alpha_{g}\left(1-\alpha_{g}\right) \rho_{g} \rho_{l}\left(v_{g}-v_{l}\right)^{2}, \\
d=\left(1-\alpha_{g}\right) \rho_{g}+\alpha_{g} \rho_{l} .
\end{gathered}
$$

We can observe that, except for the case when $v_{g}=v_{l}$, there are two complex conjugated values for the characteristic $\lambda$. Thus, since we are working in the limit $k \rightarrow \infty$, the imaginary part of omega $\omega_{I}=k \lambda_{I}$ can take arbitrary large values. Consequently, as can be seen from Eq. (11), the perturbation will grow without limit even for a small increment in time. In other words, a little disturbance of the initial state will diverge instantaneously. This is in contradiction with the third Hadamard condition for a well-posed problem because small perturbations are not reflected as small (or at least finite) changes in the solution. Therefore, the solution does not depend continuously on its data and the problem is said to be ill posed.

It is known that a well-posed problem can be guaranteed if all the characteristic values are real and distinct (strong hyperbolic system) [32, 33]. As in the one-phase case, the degeneration of the two infinite values of Eq. (15) can be removed if a finite sound velocity for each phase is considered. However, the other two complex values are not so easy to avoid. In the next section, we will show a method to solve this difficulty.

\section{PRECONDITIONING MASS MATRIX}

We have seen in the previous section that the simplest two-fluid model has complex characteristic roots and is therefore ill posed as an initial value problem except for the trivial 
case of equal phase velocities [18]. In this section, we propose a simple method (already used in one-phase flows $[38,39])$ that permits to hyperbolize the two-fluid model. This method consists in premultiplying the matrix A of Eq. (6) by another matrix M. From Eq. (6) we can see that the preconditioning matrix $\mathbf{M}$ only affects terms with temporal derivatives. Since these terms vanish when the steady state solution is achieved, this solution is not changed by the preconditioning matrix.

The preconditioning mass matrix $\mathbf{M}$ has two parameters. The first, $\beta$, only affects the inertia of the mass equation and allows the hyperbolization of the model (no complex characteristic values). The second, $\gamma$, affects each one of the temporal terms of the balance equations, its purpose being to correct the speed of propagation of the waves. Thus, the form of the proposed matrix $\mathbf{M}$ is

$$
\mathbf{M}=\gamma\left(\begin{array}{llll}
\beta & 0 & 0 & 0 \\
0 & 1 & 0 & 0 \\
0 & 0 & 1 & 0 \\
0 & 0 & 0 & 1
\end{array}\right),
$$

and defining $\tilde{\mathbf{A}}=(\mathbf{M} \mathbf{A})$, the new characteristic equation is given by

$$
\operatorname{det}(\tilde{\mathbf{A}} \lambda-\mathbf{B})=0
$$

Developing the determinant, we arrive to the general expression

$$
I_{0} \lambda^{2}+I_{1} \lambda+I_{2}=0
$$


where

$$
\begin{gathered}
I_{0}=-\left(\rho_{l} \alpha_{g}+\beta \rho_{g} \alpha_{l}\right), \\
I_{1}=2\left(\rho_{l} v_{l} \alpha_{g}+\beta \rho_{g} v_{g} \alpha_{l}\right) / \gamma, \\
I_{2}=-\left(\rho_{l} v_{l}^{2} \alpha_{g}+\rho_{g} v_{g}^{2} \alpha_{l}\right) / \gamma^{2} .
\end{gathered}
$$

The roots of the characteristic equation, Eq. (19), are

$$
\lambda_{1,2}=-\frac{I_{1}}{2 I_{0}} \pm \sqrt{\left(\frac{I_{1}}{2 I_{0}}\right)^{2}-\frac{I_{2}}{I_{0}}},
$$

and defining $C_{1}=I_{1} /\left(2 I_{0}\right)$ and $C_{2}=I_{2} / I_{0}$ with

$$
\begin{gathered}
C_{1}=-\frac{\rho_{l} v_{l} \alpha_{g}+\beta \rho_{g} v_{g} \alpha_{l}}{\gamma\left(\rho_{l} \alpha_{g}+\beta \rho_{g} \alpha_{l}\right)}, \\
C_{2}=\frac{\rho_{l} v_{l}^{2} \alpha_{g}+\rho_{g} v_{g}^{2} \alpha_{l}}{\gamma^{2}\left(\rho_{l} \alpha_{g}+\beta \rho_{g} \alpha_{l}\right)},
\end{gathered}
$$

we arrive to the following expression for the roots

$$
\lambda_{1,2}=-C_{1} \pm \sqrt{C_{1}^{2}-C_{2}} .
$$

Since we want to assure hyperbolicity, the roots should not have imaginary components and therefore we ask for 


$$
\Delta=C_{1}^{2}-C_{2} \geq 0
$$

If the discriminant is equalized to zero, $\beta_{\text {crit }}$ values are found. Calling $A=\alpha_{l} / \alpha_{g}, B=\rho_{l} / \rho_{g}$ and $C=v_{l} / v_{g}$ we obtain

$$
\Delta=C_{1}^{2}-C_{2}=-A \beta_{c r i t}^{2}+(B C(C-2)+A) \beta_{c r i t}+B=0 .
$$

After computing the $\beta_{\text {crit }}$ from the previous equation and substituting it into Eq. (26) we reach the following expression for the characteristic roots with $\beta=\beta_{\text {crit }}$,

$$
\begin{gathered}
\lambda_{1,2}=\frac{(D \pm \sqrt{E \cdot D}) v_{g}}{\left(\rho_{l} \alpha_{g}\left(\left(v_{l}-v_{g}\right)^{2}+v_{g}^{2}\right)+\rho_{g} v_{g} \alpha_{l} \pm \sqrt{E \cdot D}\right) \gamma}, \\
D=\rho_{l} v_{l}^{2} \alpha_{g}+\rho_{g} v_{g}^{2} \alpha_{l}, \\
E=\rho_{g} v_{g}^{2} \alpha_{l}+\rho_{l} \alpha_{g}\left(v_{l}+2 v_{g}\right)^{2} .
\end{gathered}
$$

In Fig. 2 we have represented the eigenvalues of the problem without preconditioning which arise as a result of a sweeping in the void fraction and velocity relations. As it can be seen, they possess imaginary components except for velocity relations $C=v_{l} / v_{g}=1$. Fig. 3 corresponds to the problem with preconditioning for $\gamma=1$. In this case, although the characteristics do not take complex value, the propagation velocity has been modified for the parameter $\beta$. Note that not only the maximum value of the real component has been modified, but there are also negative propagation velocities. 
The parameter $\beta$ that permits hyperbolizing the differential two-fluid model modifies the real part of characteristic values. Therefore, the temporal evolution of the problem is different from the original one. With the purpose of recovering the temporal behavior, the preconditioning matrix contains a parameter $\gamma$ whose determination is next developed.

From Eq. (26) for the characteristic values, we can observe that the original system, $\beta=1$ and $\gamma=1$, presents imaginary characteristics. The real part, which determines the propagation velocity of the information in the medium, is given by

$$
\operatorname{Re}\left(\lambda_{1,2}\right)=-C_{1}(\beta=1 ; \gamma=1)
$$

Parameter $\gamma$ is obtained from the relation between the characteristic for $\beta=\beta_{\text {crit }}$ and the real part $\operatorname{Re}\left(\lambda_{1,2}\right)$. Therefore

$$
\gamma_{1,2}=-\frac{\rho_{l} \alpha_{g}+\rho_{g} \alpha_{l}}{\rho_{l} v_{l} \alpha_{g}+\rho_{g} v_{g} \alpha_{l}} \frac{(D \pm \sqrt{E \cdot D}) v_{g}}{\rho_{l} \alpha_{g}\left(\left(v_{l}-v_{g}\right)^{2}+v_{g}^{2}\right)+\rho_{g} v_{g} \alpha_{l} \pm \sqrt{E \cdot D}}
$$

In Fig. 4 we can observe the characteristic values with the parameter $\gamma$ determined by the last expression. These values do not present complex components and they recover the value of the real component corresponding to the original problem without preconditioning.

\section{NUMERICAL RESULTS}

In this section we verify numerically the validity of the preconditioning method using a time marching scheme to solve as a first example a well-known problem, the so called water 
faucet, which has analytical solution. The ill-posedness of the two-fluid model without preconditioning causes in numerical implementations a tendency to develop instabilities that grow up and propagate through the domain. These instabilities are more likely to occur at high void fractions and at large density and velocity ratios. Thus, we propose another example with unfavorable initial conditions (with tendency to instabilities) that we call wave-traveling problem. Without preconditioning, even the robust numerical scheme described in the next subsection fails to solve this second example.

\section{1 - Discretization}

The implemented numerical discretization is based on a semi-implicit scheme with donor or upwind cell differencing for the convective terms. A staggered spatial nodalization is used and thus scalar variables $\alpha, \rho$ and $p$ are determined at the center of the control volumes $(j$ index), while velocity variables $v_{g}$ and $v_{l}$ are located at the edges $(j+1 / 2$ index $)$. The discretized form of Eqs. (1)-(4) are then

$$
\begin{gathered}
\frac{1}{\Delta t}\left[\left(\alpha_{g} \rho_{g}\right)_{j}^{n+1}-\left(\alpha_{g} \rho_{g}\right)_{j}^{n}\right]+\frac{1}{\Delta x}\left[\left(\hat{\alpha}_{g} \hat{\rho}_{g}\right)_{j+1 / 2}^{n}\left(v_{g}\right)_{j+1 / 2}^{n+1}-\left(\hat{\alpha}_{g} \hat{\rho}_{g}\right)_{j-1 / 2}^{n}\left(v_{g}\right)_{j-1 / 2}^{n+1}\right]=0, \\
\frac{1}{\Delta t}\left[\left(\alpha_{l} \rho_{l}\right)_{j}^{n+1}-\left(\alpha_{l} \rho_{l}\right)_{j}^{n}\right]+\frac{1}{\Delta x}\left[\left(\hat{\alpha}_{l} \hat{\rho}_{l}\right)_{j+1 / 2}^{n}\left(v_{l}\right)_{j+1 / 2}^{n+1}-\left(\hat{\alpha}_{l} \hat{\rho}_{l}\right)_{j-1 / 2}^{n}\left(v_{l}\right)_{j-1 / 2}^{n+1}\right]=0, \\
\frac{1}{\Delta t}\left(\hat{\alpha}_{g} \hat{\rho}_{g}\right)_{j+1 / 2}^{n}\left(v_{g}^{n+1}-v_{g}^{n}\right)_{j+1 / 2}+\frac{1}{\Delta x}\left(\hat{\alpha}_{g} \hat{\rho}_{g} v_{g}\right)_{j+1 / 2}^{n}\left[\left(v_{g}\right)_{j+1 / 2}^{n}-\left(v_{g}\right)_{j-1 / 2}^{n}\right] \\
=-\frac{1}{\Delta x}\left(\alpha_{g}\right)_{j+1 / 2}^{n}\left(P_{j+1}-P_{j}\right)^{n+1}+\left(\alpha_{g} \rho_{g}\right)_{j+1 / 2}^{n} g-\left(F_{I}\right)_{j+1 / 2}^{n+1}{ }_{j}
\end{gathered}
$$


and

$$
\begin{aligned}
& \frac{1}{\Delta t}\left(\hat{\alpha}_{l} \hat{\rho}_{l}\right)_{j+1 / 2}^{n}\left(v_{l}^{n+1}-v_{l}^{n}\right)_{j+1 / 2}+\frac{1}{\Delta x}\left(\hat{\alpha}_{l} \hat{\rho}_{l} v_{l}\right)_{j+1 / 2}^{n}\left[\left(v_{l}\right)_{j+1 / 2}^{n}-\left(v_{l}\right)_{j-1 / 2}^{n}\right] \\
& =-\frac{1}{\Delta x}\left(\alpha_{l}\right)_{j+1 / 2}^{n}\left(P_{j+1}-P_{j}\right)^{n+1}+\left(\alpha_{l} \rho_{l}\right)_{j+1 / 2}^{n} g-\left(F_{I}\right)_{j+1 / 2}^{n+1}
\end{aligned} .
$$

Scalar variables $\alpha$ and $\rho$ at $j+1 / 2$ are the average value between $j$ and $j+1$ and, calling $\theta$ to $\alpha$ or $\rho$, hat variables are defined as

$$
\hat{\theta}_{j+1 / 2}=\frac{1}{2}\left(\theta_{j}+\theta_{j+1}\right)+\frac{1}{2} \frac{v_{j+1 / 2}}{\left|v_{j+1 / 2}\right|}\left(\theta_{j}-\theta_{j+1}\right),
$$

where $v$ is the velocity corresponding to the phase considered.

This numerical model is the one used in the RELAP5 code for multiphase flows. More details, like the implementation of an automatic control of the time step based on the Courant number, can be found in Refs. [28] and [40]. The inclusion of the preconditioning matrix $\mathbf{M}$ within this scheme does not present difficulties. It is just to multiply the corresponding terms in the balance equations by the parameters $\beta$ and $\gamma$ according to Eq. (17). In order to ensure strong hyperbolicity (real and distinct characteristic values), large but finite sound velocities as well as $\beta$ parameters slightly greater than $\beta_{\text {crit }}$ were used.

\section{2 - Water faucet problem}

Due to the fact that it has analytical solution, the water faucet problem devised by Ransom [41] is widely used to validate two-phase flow models [42-44]. The problem consists of a 
$12 \mathrm{~m}$ long vertical tube where there is initially a uniform volume fraction $\left(\alpha_{l}^{0}=0.6\right)$ of water $\left(\rho_{l}=1000 \mathrm{~kg} / \mathrm{m} 3\right)$ that is moving at constant velocity $\left(v_{l}^{0}=15 \mathrm{~m} / \mathrm{s}\right)$ in a stagnant air annulus. No interaction between phases is considered. When the simulation starts $(t=0)$, a gravity field $\left(\mathrm{g}=9.8 \mathrm{~m} / \mathrm{s}^{2}\right)$ is applied and this causes the water column to accelerate. At the top of the tube (inlet), water volume fraction and velocity are kept unchanged $\left(\alpha_{l}^{\text {inlet }}=\alpha_{l}^{0}\right.$ and $v_{l}^{\text {inlet }}=v_{l}^{0}$ ), and at the bottom (outlet) a constant pressure is maintained ( $p^{\text {outlet }}=10^{5} \mathrm{~Pa}$ ). Due to the acceleration, a contact discontinuity propagates downwards until a steady state is reached when the discontinuity arrives at the outlet.

Neglecting pressure gradient in both fluids, the analytical solution to the water faucet problem is given by:

$$
\text { If } x \leq v_{l}^{0} t+\frac{1}{2} g t^{2}\left\{\begin{array}{l}
\alpha_{g}=1-\frac{\alpha_{l}^{0} v_{l}^{0}}{v_{l}} \\
v_{l}=\left(\left(v_{l}^{0}\right)^{2}+2 g x\right)^{1 / 2}
\end{array} ; \text { and otherwise } \alpha_{g}=1-\alpha_{l}^{0}\right.
$$

Using the preconditioning mass matrix, this problem was simulated with six different meshes of 40, 80, 160, 320, 640 and 1280 uniform lineal elements. In Figs. 5 and 6 numerical as well as analytical solutions for the void fraction and water velocity are shown. These figures correspond to a time $(\mathrm{t}>2 \mathrm{~s})$ in which the steady state was reached. It can be seen that, as mentioned above, the preconditioning method does not affect the steady state.

Figures 7 and 8 show the void fraction and liquid velocity respectively, at time $(t=0.4 \mathrm{~s})$. At this time, the discontinuity is still within the tube. Although agreement between numerical and analytical solutions in a transient state is not expected (we are using $\gamma=1$ ), these figures 
show that the velocity of propagation of the discontinuity is well reproduced. Besides, numerical solutions for water velocity and void fraction approach analytical values when the number of elements is increased, capturing the contact discontinuity very well.

\section{3 - Wave traveling problem}

In this problem, unfavorable conditions are set up in order to reflect in the numerical simulation the ill-posed character of the differential equations. The domain is onedimensional and its length $\mathrm{L}=0.4 \mathrm{~m}$ is discretized in 100 elements. The density ratio $\rho_{l} / \rho_{g}$ is 1000:1 and all the fields are initially uniform $\left(v_{l}=1 \mathrm{~m} / \mathrm{s}, v_{g}=10 \mathrm{~m} / \mathrm{s}, p=0\right)$ except the void fraction that is a sinusoidal perturbation $\left(\alpha_{\mathrm{g}}=0.5+0.45 \sin (4 \pi x / L)\right)$. Periodic boundary conditions are imposed. Thus, waves generated by the void fraction perturbation can propagate freely through the domain. As in the water faucet problem, no interaction between phases is considered in this case. Although the initial conditions can seem rather extreme, these conditions (or even worse ones) appear for example in the steelmaking industry when argon is injected at the bottom of the ladle to produce the stirring of the liquid steel.

Figure 9 shows the void fraction obtained without preconditioning right before the numerical implementation becomes unstable at 5 time steps of $0.001 \mathrm{~s}$. This instability is more evident in Fig. 10, where the gas velocity is plotted. Figs. 11 and 12 were obtained with preconditioning and they correspond to 5 time steps of $0.001 \mathrm{~s}$. As can be observed, using the preconditioning method the solution does not diverge and is smooth. This is not surprising since now we are working with a well-posed system of differential equations. The simulation can be continued and no sign of instability is found as can be seen in Figs. 13 and 14 where 
we show the same curves after 25 time steps of 0.001 . It is also worth to note that the same problem has been solved with the commercial code CFX [45], and the solution diverges after 7 time steps of $0.001 \mathrm{~s}$.

\section{CONCLUSIONS}

We have developed a preconditioning method for the mass matrix of the two-fluid model in order to make the model well-posed. The preconditioning matrix has two parameters: the first one $\beta$ is for making the model hyperbolic, and the second one $\gamma$ is for recovering the wave velocity propagation corresponding to the original model. Both parameters depend on the void fraction and on velocity and density relations between phases.

The characteristic values obtained with the preconditioning method do not show any imaginary component and share the same real part with the original model. To test the proposed method we have modified a well-established numerical scheme to include the preconditioning. Two examples were analyzed using $\gamma=1$. One of them, the so called water faucet problem, is a well known benchmark widely used for two-phase flows. Using the preconditioning method, the numerically obtained steady state solution agrees with the analytical solution. Besides, although the time evolution cannot be guaranteed using $\gamma=1$, the method reproduces transient solutions very well. The second example was chosen in order to evidence numerically the ill-posed nature of the two-fluid model. This example, a large amplitude traveling wave, allowed us to see how the ill-posedness is reflected as instabilities in the numerical simulation and shows that, as expected, these instabilities do not appear when the differential equations are made well-posed by means of the preconditioning matrix. 


\section{REFERENCES}

[1] Ishii, M., 1975, Thermofluid Dynamic Theory of Two-phase Flow, Eyrolles, Paris.

[2] Hirt, C. W., and Nichols, B. D., 1981, "Volume of Fluid (VOF) Method for the Dynamics of Free Boundaries,” Journal of Computational Physics, 39, pp. 201-225.

[3] Kothe, D. B., and Mjolsness R. C., 1992, "RIPPLE: A New Model for Incompressible Flows with Free Surfaces,” AIAA Journal, 30, pp. 2694-2700.

[4] Kothe, D. B., Rider, W. J., Mosso, S. J., Brock, J. I., and Hochstein, J. S., 1996, "Volume Tracking of Interfaces having Surface Tension in Two and Three Dimensions," Technical Report AIAA 96-0859, AIAA.

[5] Richards, J. R., Beris, A. N., and Lenho, A. M., 1995, "Drop Formation in LiquidLiquid Systems Before and After Jetting,” Phys. Fluids, 7, pp. 2617- 2630.

[6] Rider, W. J., Kothe, D. B., Mosso, S. J., Cerutti, J., and Hochstein, J. S., 1995, "Volume Tracking of Interfaces having Surface Tension in Two and Three dimensions," Technical Report AIAA 95-0699, AIAA.

[7] Rider, W. J., and Kothe, D. B., 1998, "Reconstructing Volume Tracking," Journal of Computational Physics, 141, pp. 112-152.

[8] Juric, D., and Tryggvason, G., 1998, "Computations of Boiling Flows," Int. J. Multiphase Flow, 24, pp. 387-410.

[9] Unverdi, S. O., and Tryggvason, G., 1992, “A Front-Tracking Method for Viscous Incompressible Multi-Fluid Flows," Journal of Computational Physics, 100, pp. 25-37. 
[10] Fedkiw, R. P., Aslam, T., Merriman, B., and Osher, S., 1999, “A Non-Oscillatory Eulerian Approach to Interfaces in Multimaterial Flows (the Ghost Fluid Method)," Journal of Computational Physics, 152, (2), pp. 457-492.

[11] Osher, S., and Sethian, J. A., 1988, "Fronts Propagating with Curvature Dependent Speed: Algorithm Based on Hamilton-Jacobi Formulations," Journal of Computational Physics, 79, pp. 12-49.

[12] Osher, S., and Fedkiw, R. P., 2001, "Level Set Methods: An Overview and Some Recent Results,” Journal of Computational Physics, 169, pp. 463-502.

[13] Sussman, M., and Smereka, P., 1997 “Axisymmetric Free Boundary Problems,” J. Fluid Mech., 341, pp. 269-294.

[14] Anderson, D. M., McFadden, G. B., and Wheeler, A. A., 1998, "Diffuse Interface Methods in Fluid Mechanics,” Ann. Rev. Fluid Mech., 30, pp. 139-165.

[15] Jacqmin, D., 1999, “Calculation of Two-Phase Navier-Stokes Flows Using Phase-Field Modeling,” J. Comp. Phys., 155, pp. 96-127.

[16] Lowengrub, J., and Truskinovsky, L., 1998, "Quasi-Incompressible Cahn-Hilliard Fluids and Topological Transitions,” In: Proc. R. Soc. London Ser. A 454, pp. 26172654.

[17] Harlow, F. H., and Welch, J. E., 1965, "Numerical Calculation of Time-Dependent Viscous Incompressible Flow of Fluids with A Free Surface,” Physics of Fluids, 8, pp. $2182-2189$.

[18] Drew, D. A., and Passman, S. L., 1998, Theory of Multicomponent Fluids, Applied Mathematical Sciences 135, SpringerVerlag, New York, Berlin, Heidlberg. 
[19] Keyfitz, B. L., 2000, "Mathematical Properties of Nonhyperbolic Models for Incompressible Two-Phase Flow," Proceedings of the ICMF 2001.

[20] Joseph, D. D., and Saut, J. C., 1990, “Short Wave Instabilities and Ill Posed Initial Value Problems," Journal of Theoretical and Computational Fluid Mechanics, 1, pp.191-227.

[21] Méndez, C. G., Nigro, N., and Cardona, A., 2005, "Drag and Non-Drag Force Influences in Numerical Simulations of Metallurgical Ladles," Journal of Materials Processing Technology, 160, pp. 296-305.

[22] Lahey, R. T. Jr., Yin, J., and Tiwari, P., 2005, “Analysis of Void Wave Propagation and Sonic Velocity Using a Two-Fluid Model,” Multiphase Science and Technology, 17, pp. 293-320.

[23] Chung, M., Chang, K., and Lee, S., 2002, "Numerical Solution of Hyperbolic TwoFluid Two-Phase Flow Model with Non-Reflecting Boundary Conditions,” Int. Journal of Engineering Science, 40, pp. 789-803.

[24] Micaelli, J. C., 1988, Document Syntheses CATHARE (CATHARE An Advanced BestEstimate Code for PWR Safety Analysis), SETh/LEML-EM/88-129, Grenoble Cedex.

[25] Bestion, D., 1990, "The Physical Closure Laws in CATHARE Code," Nucl. Eng. Design, 124, 229-245.

[26] Coquel, F., Gallouët, T., Hérard, J., and Seguin, N., 2002, "Closure Laws for a TwoFluid Two-Pressure Model,” C.R. Acad. Sci. Paris, I-334 , pp. 927-932. 
[27] Lee, S., Chang, K., and Kim, K., 1998, "Pressure Wave Speeds from the Characteristics of Two Fluids, Two-Phase Hyperbolic Equation System," Int. Journal of Multiphase Flow, 24, pp. 855-866.

[28] Pokharna, H., Mori, M., and Ransom, V. H., 1997, "Regularization of Two-Phase Flow Models: A Comparison of Numerical and Differential Approaches”, J. Comp. Phys. 134, pp. 282-295.

[29] Städtke, H., Franchello, G., and Worth, B., 1997, "Numerical Simulation of MultiDimensional Two-Phase Flow Based on Flux Vector Splitting," Nuclear Engineering and Design, 177, pp. 199-213.

[30] Städtke, H., and Holtbecker, R., "Hyperbolic model for one-dimensional inhomogeneous two-phase flow," 29th Meet. Of European Two-Phase Flow Group, June 2-3, Stockholm, Sweden.

[31] Anderson, A., and York, J., 1999, “Fixing Einstein's Equations,” Phys. Rev. Let., 82, pp. 43-84.

[32] Reula O., 2004, "Strongly Hyperbolic Systems," General Relativity and Quantum Cosmology, e-print 0403007.

[33] Gundlach C. and Martin-García J., 2005, "Hyperbolicity of Second Order in Space Systems of Evolution Equations," General Relativity and Quantum Cosmology, e-print 0506037.

[34] Jones, A. V., and Prosperetti, A., 1985, "On the Stability of First-Order Differential Models for Two-Phase Flow Prediction,” Int. J. Multiphase Flow, 11, pp. 133-148. 
[35] Zhang, D. Z., and Prosperetti, A., 1994, "Ensemble Phase Averaged Equations for Bubbly Flows," Phys. Fluids, 6, pp. 295-629.

[36] Lahey, R. T. Jr., and Drew, D. A., 1988, “The Three-Dimensional Time and Volume Averaged Conservation Equations of Two-Phase Flows,” Adv. Nucl. Sci. Technol, 20, pp. 1-69.

[37] Ishii, M., and Zuber, N., 1979, "Relative Motion and Interfacial Drag Coefficient in Dispersed Two-Phase Flow of Bubbles, Drops and Particles,” AIChE J., 25, pp. 843855.

[38] Chorin, A. J., 1967, “A Numerical Method for Solving Incompressible Viscous Problems", J. Comp. Phys., 2, pp. 12-26.

[39] Turkel, E., 1999, "Preconditioning Techniques in Computational Fluid Dynamics", Annual Review of Fluid Mechanics, 31, pp. 385-416.

[40] Ransom, V. H. et al. 1985, RELAP5/MOD2 Code Manual 1, Code 2, Structure, System Models, and Solution Methods, NUREG/CR-4312.

[41] Shieh, A. S., Ransom, V. H. and Krishnamurthy, R., 1994, RELAP5/MOD3 Code Manual Volume 6, Validation of Numerical Techniques in RELAP5/MOD3.0, NUREG/CR-5535.

[42] Coquel, F., Amine, K. El., Godlewski, E., Perthame, B., Rascle, P., 1997, “A Numerical Method Using Upwind Schemes for the Resolution of Two-Phase Flows”, J. Comput. Phys., 136, pp. 272-288. 
[43] Niu, Y.Y., 2001, “Advection Upwinding Splitting Method to Solve a Compressible Two-Fluid Model,” Int. J. Numer. Meth. Fluids, 36, 351-371.

[44] Trapp, J.A. and Riemke, R.A., 1986,“A Nearly-Implicit Hydrodynamic Numerical Scheme for Two-Phase Flows," J. Comput. Phys., 66, 62-82.

[45] AEA Technology. User Manual to CFX Version 5.7. AEA Technology plc, Harwell, UK, (2004). 


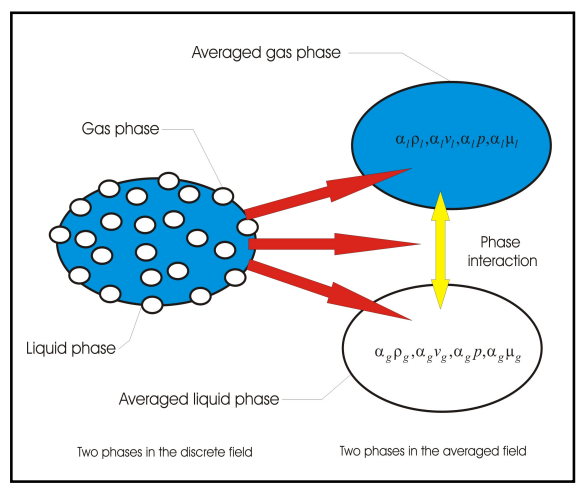

Figure 1. Schematic representation of two-fluid model.

JAM-05-1230; Zanotti, Méndez, Nigro and Storti; [29]. 


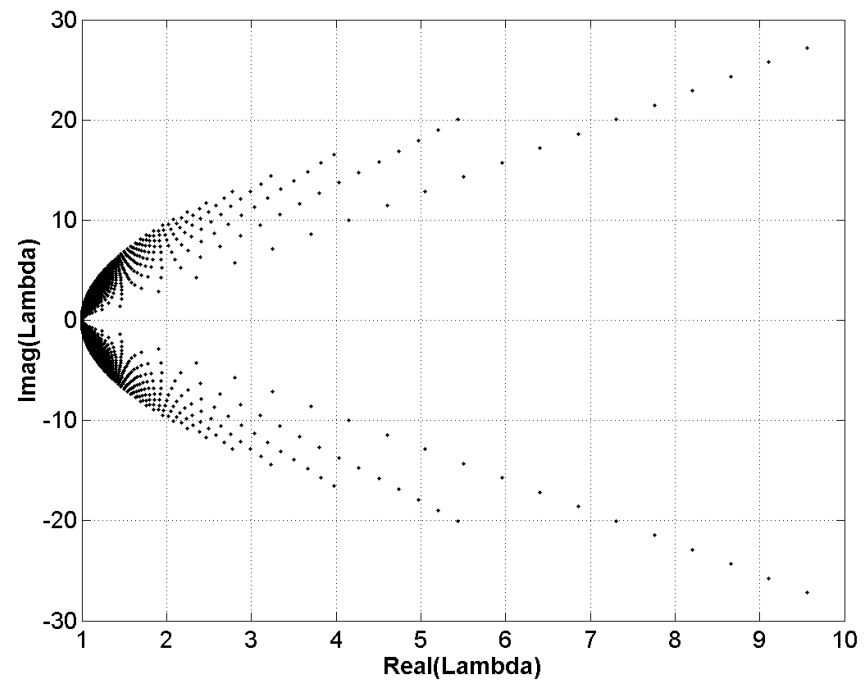

Figure 2. Eigenvalues for a sweeping in alpha (0.01:0.01:0.99) and velocity relations (1:5:100), without preconditioning.

JAM-05-1230; Zanotti, Méndez, Nigro and Storti; [30]. 


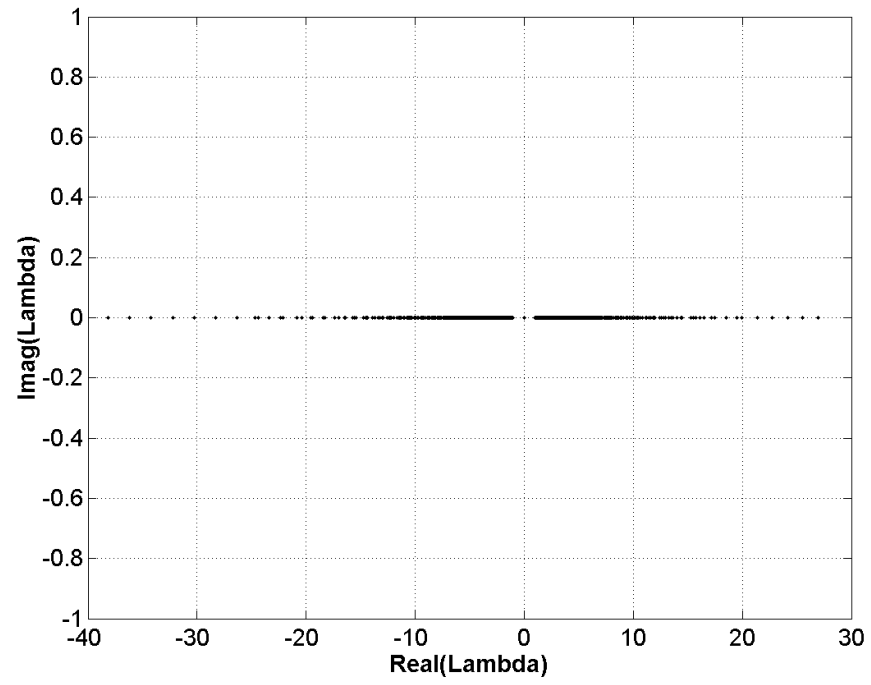

Figure 3. Eigenvalues for a sweeping in alpha (0.01:0.01:0.99) and velocity relations (1:5:100), with preconditioning and $\gamma=1$. All eigenvalues are real, but are different from the real part of eigenvalues of Fig. 2 . 


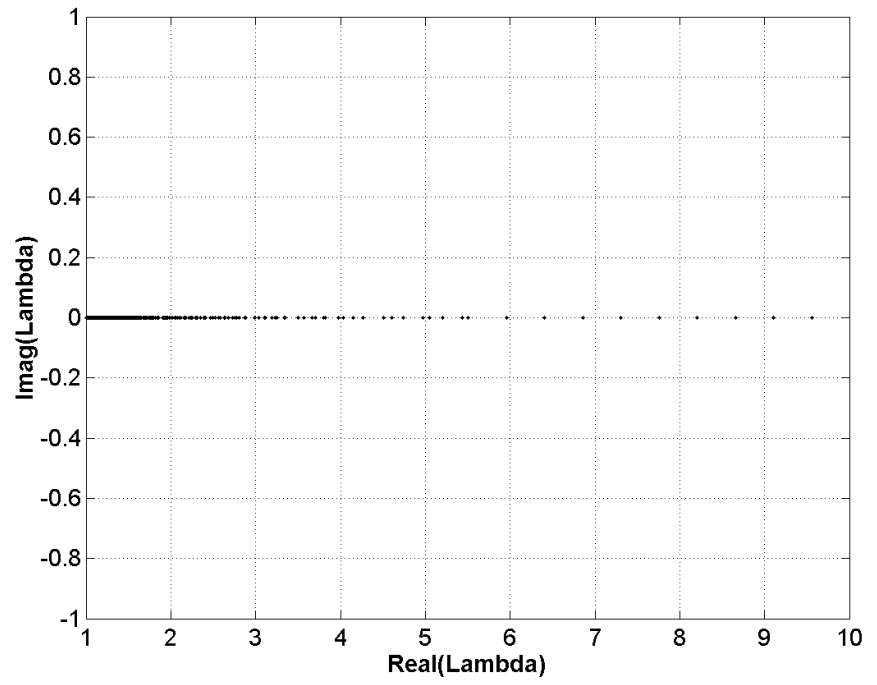

Figure 4. Eigenvalues for a sweeping in alpha (0.01:0.01:0.99) and velocity relations (1:5:100) with preconditioning and $\gamma \neq 1$. All eigenvalues are real and are equal to the real part of eigenvalues of Fig. 2.

JAM-05-1230; Zanotti, Méndez, Nigro and Storti; [32]. 


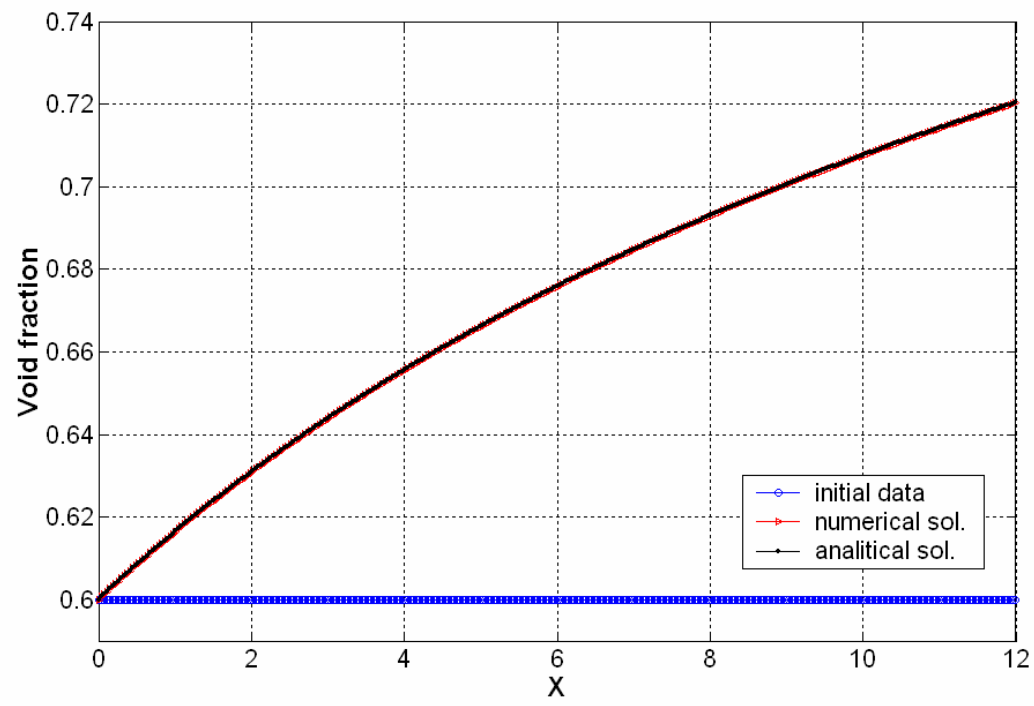

Figure 5. The water faucet problem. Void fraction with preconditioning at steady state, for a mesh of 320 uniform lineal elements. Comparison between numerical and analytical solutions.

JAM-05-1230; Zanotti, Méndez, Nigro and Storti; [33]. 


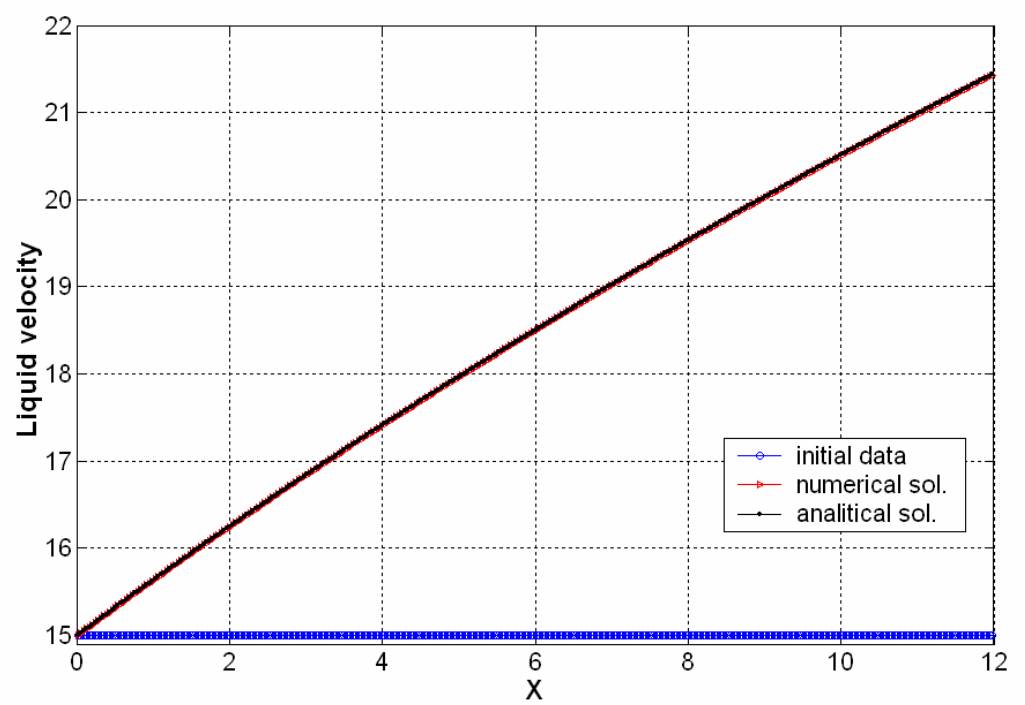

Figure 6. The water faucet problem. Liquid velocity with preconditioning at steady state, for a mesh of 320 uniform lineal elements. Comparison between numerical and analytical solutions.

JAM-05-1230; Zanotti, Méndez, Nigro and Storti; [34]. 


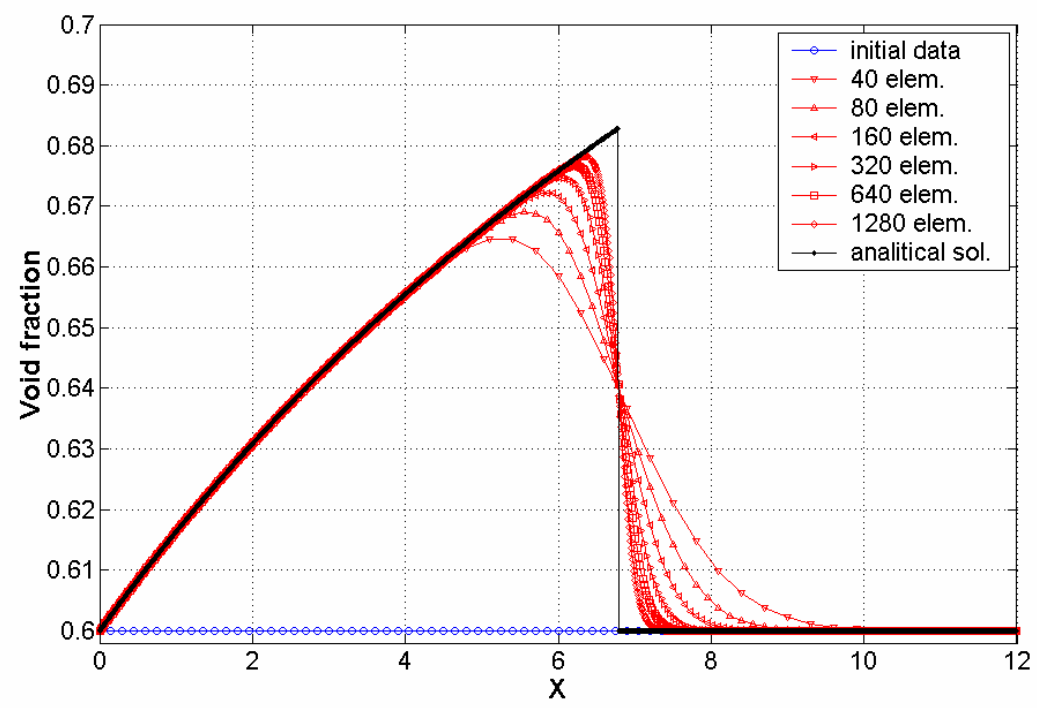

Figure 7. The water faucet problem. Void fraction with preconditioning at $\mathrm{t}=0.4 \mathrm{~s}$, for six different meshes of 40 , $80,160,320,640$ and 1280 uniform lineal elements.

JAM-05-1230; Zanotti, Méndez, Nigro and Storti; [35]. 


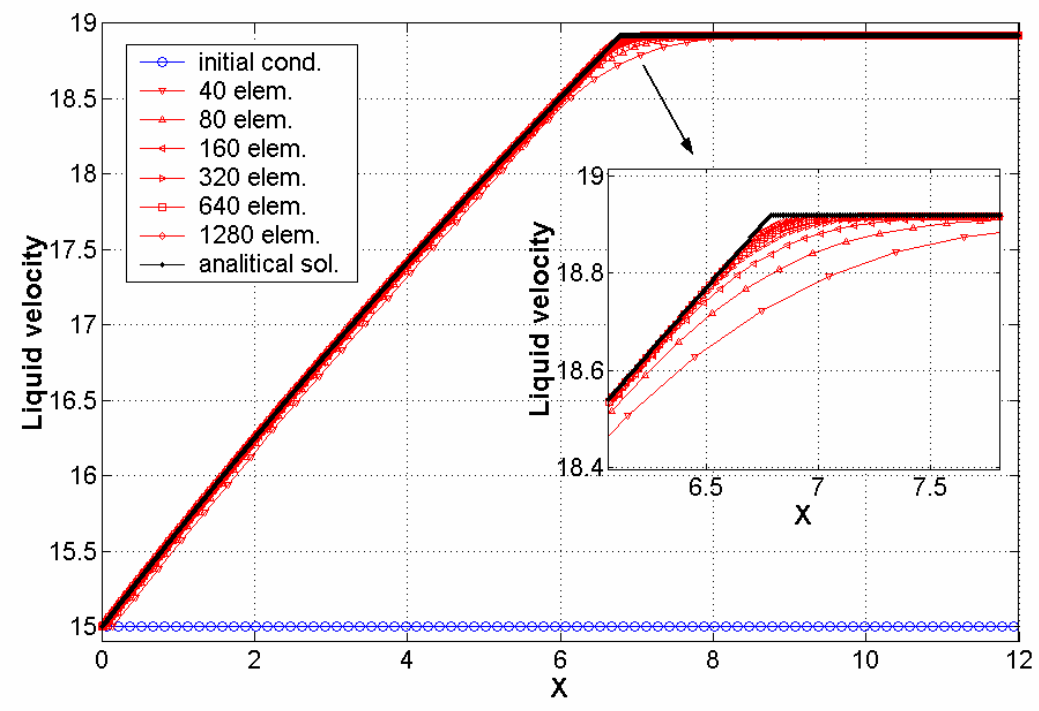

Figure 8 . The water faucet problem. Liquid velocity with preconditioning at $\mathrm{t}=0.4 \mathrm{~s}$, for six different meshes of $40,80,160,320,640$ and 1280 uniform lineal elements.

JAM-05-1230; Zanotti, Méndez, Nigro and Storti; [36]. 


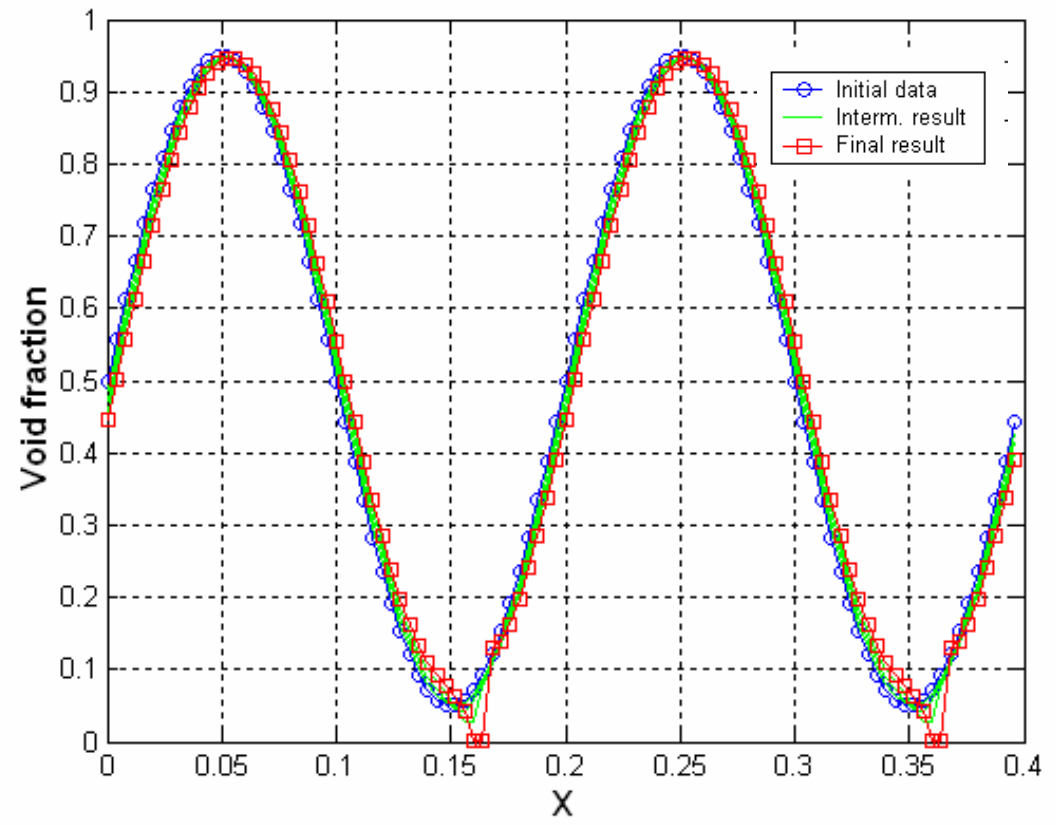

Figure 9. The wave traveling problem. Void fraction without preconditioning at 4 time steps of $0.001 \mathrm{~s}$. 


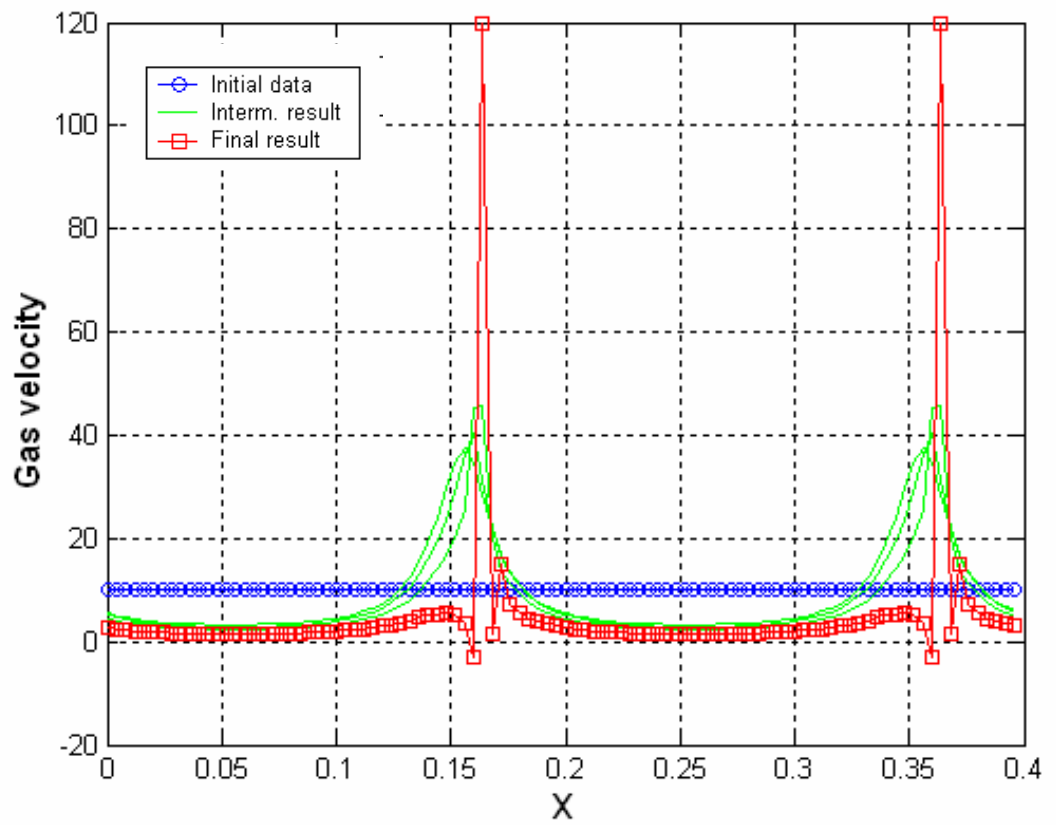

Figure 10. The wave traveling problem. Gas velocity without preconditioning at 4 time steps of $0.001 \mathrm{~s}$.

JAM-05-1230; Zanotti, Méndez, Nigro and Storti; [38]. 


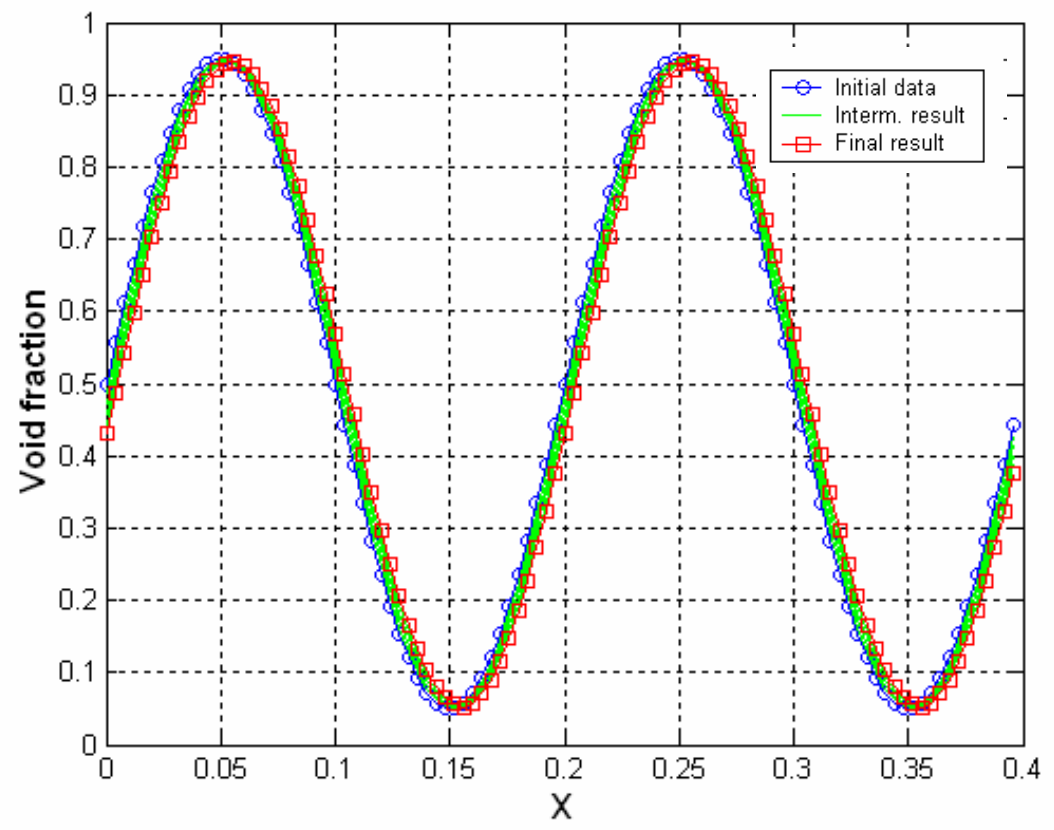

Figure 11. The wave traveling problem. Void fraction with preconditioning at 5 time steps of $0.001 \mathrm{~s}$. 


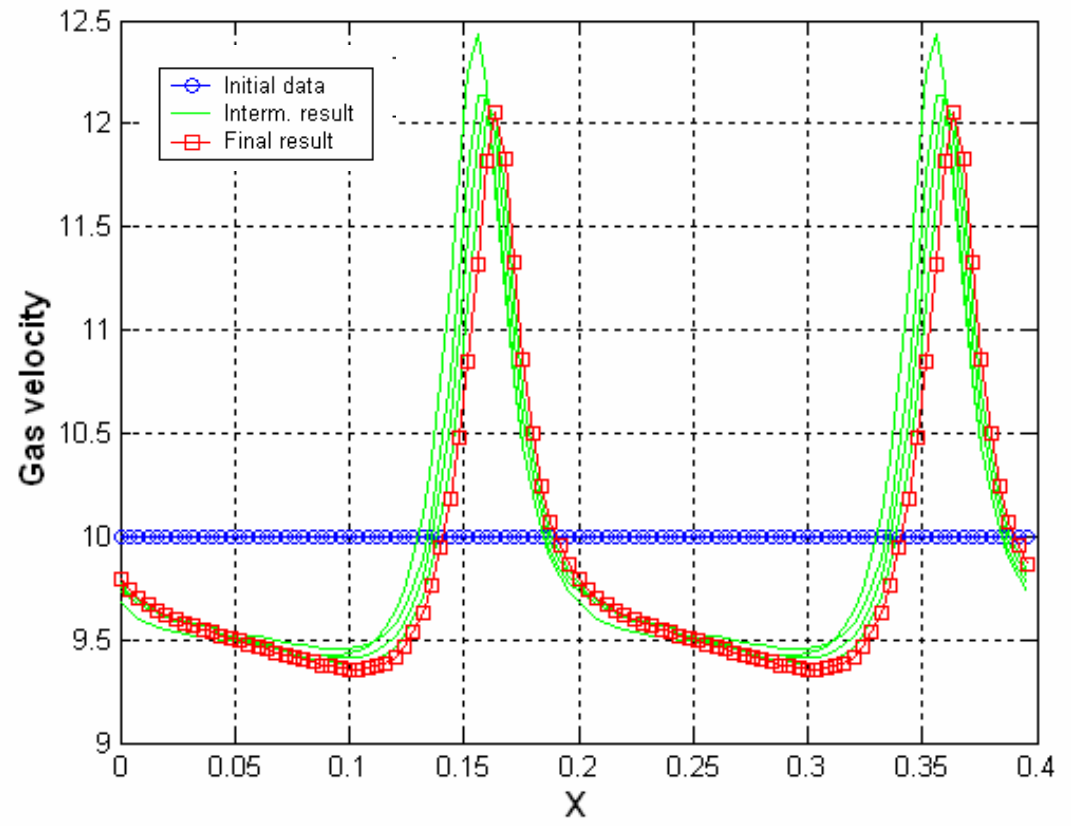

Figure 12. The wave traveling problem. Gas velocity with preconditioning at 5 time steps of $0.001 \mathrm{~s}$.

JAM-05-1230; Zanotti, Méndez, Nigro and Storti; [40]. 


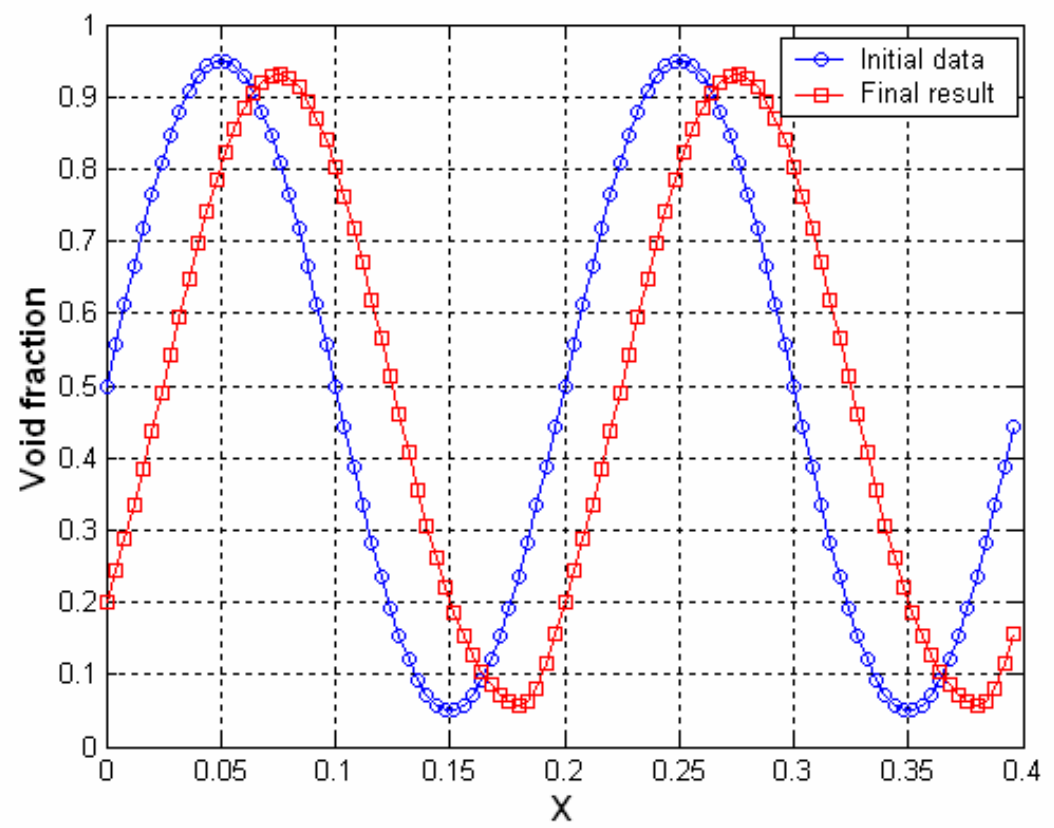

Figure 13. The wave traveling problem. Void fraction with preconditioning at 25 time steps of $0.001 \mathrm{~s}$. 


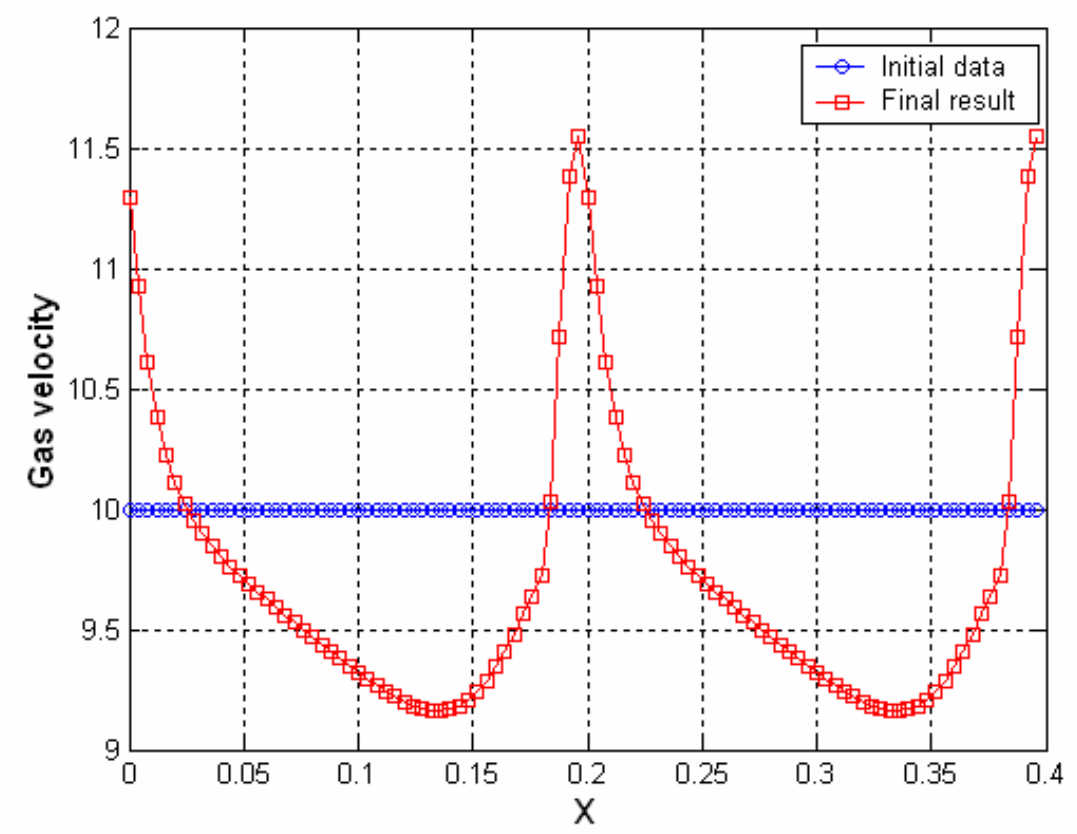

Figure 14. The wave traveling problem. Gas velocity with preconditioning at 25 time steps of $0.001 \mathrm{~s}$. 


\section{List of Figures}

1 Schematic representation of two-fluid model.

2 Eigenvalues for a sweeping in alpha (0.01:0.01:0.99) and velocity relations (1:5:100), without preconditioning.

3 Eigenvalues for a sweeping in alpha (0.01:0.01:0.99) and velocity relations (1:5:100), with preconditioning and $\gamma=1$. All eigenvalues are real, but are different from the real part of eigenvalues of Fig. 2.

4 Eigenvalue for a sweeping in alpha (0.01:0.01:0.99) and velocity relations (1:5:100) with preconditioning and $\gamma \neq 1$. All eigenvalues are real and are equal to the real part of eigenvalues of Fig. 2

5 The water faucet problem. Void fraction with preconditioning at steady state, for a mesh of 320 uniform lineal elements. Comparison between numerical and analytical solutions.

6 The water faucet problem. Liquid velocity with preconditioning at steady state, for a mesh of 320 uniform lineal elements. Comparison between numerical and analytical solutions

7 The water faucet problem. Void fraction with preconditioning at $\mathrm{t}=0.4 \mathrm{~s}$, for six different meshes of 40 , $80,160,320,640$ and 1280 uniform lineal elements.

8 The water faucet problem. Liquid velocity with preconditioning at $t=0.4 \mathrm{~s}$, for six different meshes of 40, 80, 160, 320, 640 and 1280 uniform lineal elements

9 The wave traveling problem. Void fraction without preconditioning at 4 time steps of 0.001 s....... 37

10 The wave traveling problem. Gas velocity without preconditioning at 4 time steps of 0.001 s........ 38

11 The wave traveling problem. Void fraction with preconditioning at 5 time steps of $0.001 \mathrm{~s}$ 
12 The wave traveling problem. Gas velocity with preconditioning at 5 time steps of $0.001 \mathrm{~s}$

13 The wave traveling problem. Void fraction with preconditioning at 25 time steps of $0.001 \mathrm{~s}$

14 The wave traveling problem. Gas velocity with preconditioning at 25 time steps of 0.001 